\title{
Improvement of Solar and Wind forecasting in southern Italy through a multi-model approach: preliminary results
}

\author{
Elenio Avolio ${ }^{1}$, Rosa Claudia Torcasio ${ }^{1}$, Teresa Lo Feudo ${ }^{1}$, Claudia Roberta Calidonna ${ }^{1}$, \\ Daniele Contini ${ }^{2}$, and Stefano Federico ${ }^{3}$ \\ ${ }^{1}$ Institute of Atmospheric Sciences and Climate - Italian National Research Council (ISAC-CNR), \\ Lamezia Terme, 88046, Italy \\ ${ }^{2}$ Institute of Atmospheric Sciences and Climate - Italian National Research Council (ISAC-CNR), \\ Lecce, 73100, Italy \\ ${ }^{3}$ Institute of Atmospheric Sciences and Climate - Italian National Research Council (ISAC-CNR), \\ Rome, 00133, Italy
}

Correspondence to: Elenio Avolio (e.avolio@ isac.cnr.it)

Received: 14 January 2016 - Revised: 10 April 2016 - Accepted: 13 April 2016 - Published: 20 April 2016

\begin{abstract}
The improvement of the Solar and Wind short-term forecasting represents a critical goal for the weather prediction community and is of great importance for a better estimation of power production from solar and wind farms.

In this work we analyze the performance of two deterministic models operational at ISAC-CNR for the prediction of short-wave irradiance and wind speed, at two experimental sites in southern Italy.

A post-processing technique, i.e the multi-model, is adopted to improve the performance of the two mesoscale models.

The results show that the multi-model approach produces a significant error reduction with respect to the forecast of each model. The error is reduced up to $20 \%$ of the model errors, depending on the parameter and forecasting time.
\end{abstract}

\section{Introduction}

Solar and wind farm power prediction is of great importance for renewable energy applications (Giebel et al., 2011; Monteiro et al., 2009; Pinson et al., 2009). The models for power prediction often need the output of numerical weather prediction models. Hence, the quality of the power forecast is strictly related to the quality of the radiation and wind prediction over the considered area (Alessandrini et al., 2013; Pinson et al., 2007; Von Bremen, 2007).

A good estimation of power production from solar and wind farms is a significant issue in Southern Italy, due to a large availability of solar and wind energy in spite of a poor integration into the grid (GSE, 2015).

The purpose of this work is to analyse the performance of two deterministic atmospheric models: the WRF (Weather Research and Forecasting Model) (Skamarock et al., 2008) and the RAMS (Regional Atmospheric Modeling System) (Cotton et al., 2003). Both models were run for six months of 2013 (summer and fall) at $4 \mathrm{~km}$ horizontal resolution over Italy. Verification is conducted against two surface stations located in Lamezia Terme and Lecce, and are based on hourly forecasts output and observations.

A multi-model approach is adopted to reduce the forecast errors. The multi-model is trained using a dataset of past forecasts and observations to compute the weights of the models that minimize the Root Mean Square Error; in the forecast phase these weights are used to determine the best estimate of the forecast.

\section{Methodology}

The models considered in this work are the WRF (Weather Research and Forecasting Model) and the RAMS (Regional 


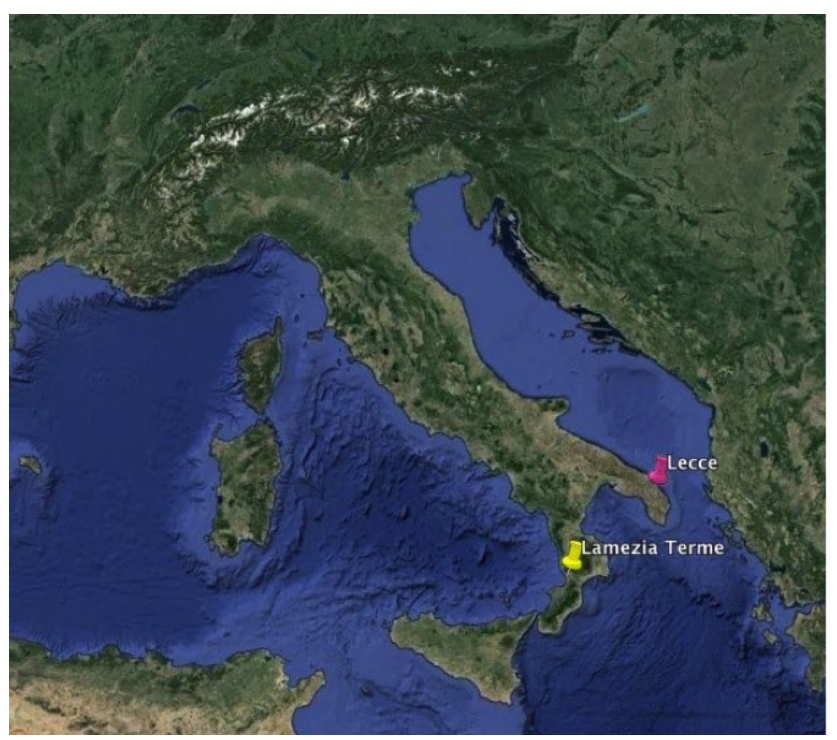

Figure 1. WRF/RAMS domain (same grid at $4 \mathrm{~km}$ resolution), and location of the experimental sites.

Atmospheric Modeling System). They were run for 6 months (from 1 June to 30 November 2013) at $4 \mathrm{~km}$ horizontal resolution over Italy, using the same domain (Fig. 1). Initial and dynamic boundary conditions are given by the 12:00 UTC deterministic analysis/forecast cycle of the ECMWF-IFS model $\left(0.25^{\circ}\right.$ horizontal latitude-longitude grid spacing).

For the WRF model, the PBL scheme adopted is the Mellor-Yamada-Janjic (MYJ) (Janjić, 1994), a 1.5-order prognostic TKE scheme with local vertical mixing. For the shortwave radiation, the Goddard scheme (Chou and Suarez, 1999) is used.

For the RAMS model, the radiation scheme detailed in Chen and Cotton (1983) is used for short-wave radiation; this scheme accounts for the total condensate present in the atmosphere but not for the specific phase of water (i. e. vapour, liquid or ice). The scheme takes into account for the ozone and carbon dioxide on radiative transfer. Unresolved vertical transport is parameterized by the $\mathrm{K}$ theory, in which the covariance is evaluated as the product of an eddy mixing coefficient and the gradient of the transported quantity. The turbulent mixing in the horizontal directions is parameterized following Smagorinsky (1963).

Each forecast lasts $36 \mathrm{~h}$ and the first $12 \mathrm{~h}$ are not considered for the comparison (spin-up time); thus the forecasttime verification is $24 \mathrm{~h}$. Short-wave radiation and $10 \mathrm{~m}$ wind speed are simulated and verified.

Verification is carried out against two surface stations located in Southern Italy, Lamezia Terme and Lecce, and are based on hourly output of models forecast; the forecasts are interpolated bilinearly to the positions of the surface stations. Experimental sites are shown in Fig. 1.
Table 1. RMSE, MAE and BIAS for Lecce and Lamezia Terme and for each model. Statistics are computed starting from hourly data for the six-month period summer-fall 2013.

\begin{tabular}{lcccc}
\hline & $\begin{array}{c}\text { Shortwave RAD } \\
{\left[\mathrm{W} \mathrm{m}^{-2}\right]}\end{array}$ & $\begin{array}{c}\text { Wind Speed } \\
{\left[\mathrm{m} \mathrm{s}^{-1}\right]}\end{array}$ \\
\hline LECCE & RAMS & WRF & RAMS & WRF \\
\hline BIA & 18 & 41 & 1.3 & 2.9 \\
MAE & 47 & 53 & 1.4 & 2.9 \\
RMSE & 96 & 104 & 1.6 & 3.4 \\
\hline LAMEZIA T & & & & \\
\hline BIA & 17 & 45 & 0.1 & 0.4 \\
MAE & 49 & 54 & 1.0 & 1.1 \\
RMSE & 99 & 105 & 1.3 & 1.4 \\
\hline
\end{tabular}

The two independent forecasts are regressed towards the measured data during the training period, defining the coefficient of the linear regression to be used in the multi-model forecast phase. More specifically, the two-model forecast at each station location is given by:

$S=\bar{O}+\sum_{i=1}^{N} a_{i}\left(F_{i}-\bar{F}_{i}\right)$

where $N$ is the number of the models (2); $a_{i}$, the weight of the $i$ th model; $F_{i}$, the forecast of the $i$ th model; $\bar{F}_{i}$, its mean value over the training period; and $\bar{O}$, the mean observation over the training period. The calculation of the weights $a_{i}$ is given by the minimization of the mean square distance $D^{2}$ :

$D^{2}=\sum_{k=1}^{L}\left(S_{k}-O_{k}\right)^{2}$

where $L$ is the training period length ( $80 \%$ of the data).

Therefore, the available dataset is divided in two parts: a training period containing $80 \%$ of the data and a forecasting period with the remaining $20 \%$. The weights $a_{i}$ are evaluated over the training period and are then used to compute the forecast ("cross-evaluation"). The methodology is applied 20 times randomly selecting the training dataset to assess the statistical robustness of the results.

\section{Results and discussions}

First of all, we have computed error statistics for each model, for the two parameters and for both experimental sites. As a second step, the multi-model technique is adopted to improve the forecast performance. Statistics used to quantify the performance of the models are: RMSE (Root Mean Square Error), MAE (Mean Absolute Error) and BIA (Bias). 


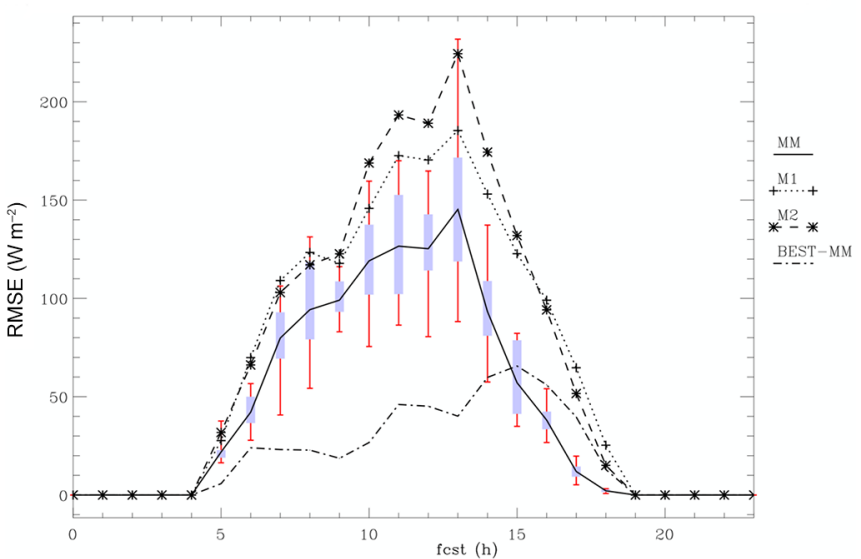

(a)

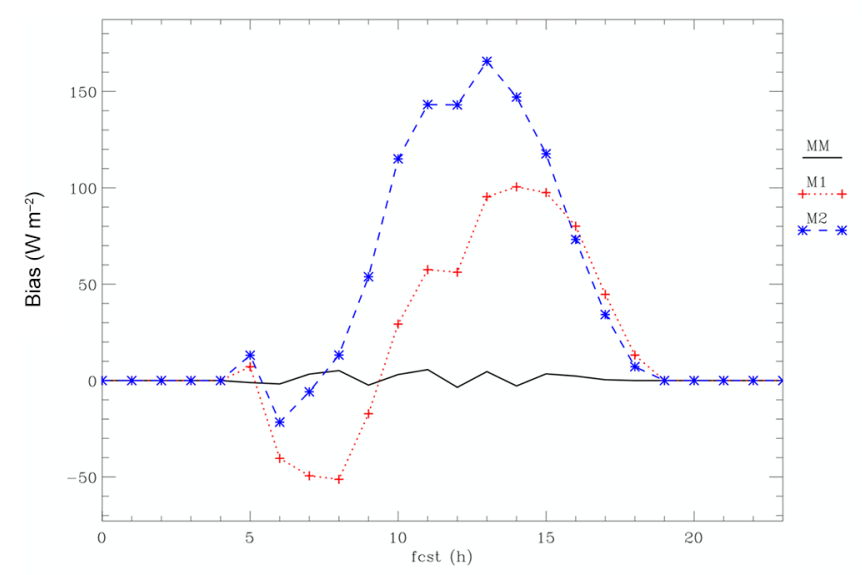

(c)

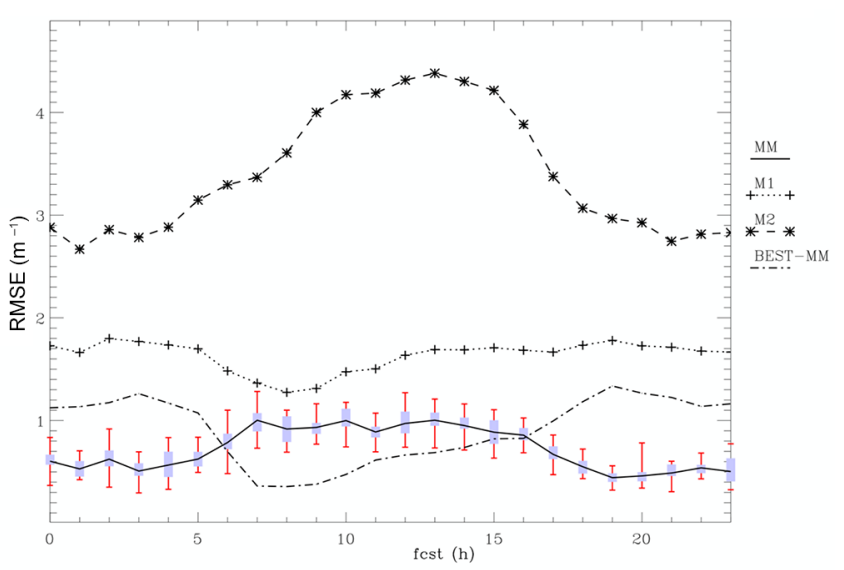

(b)

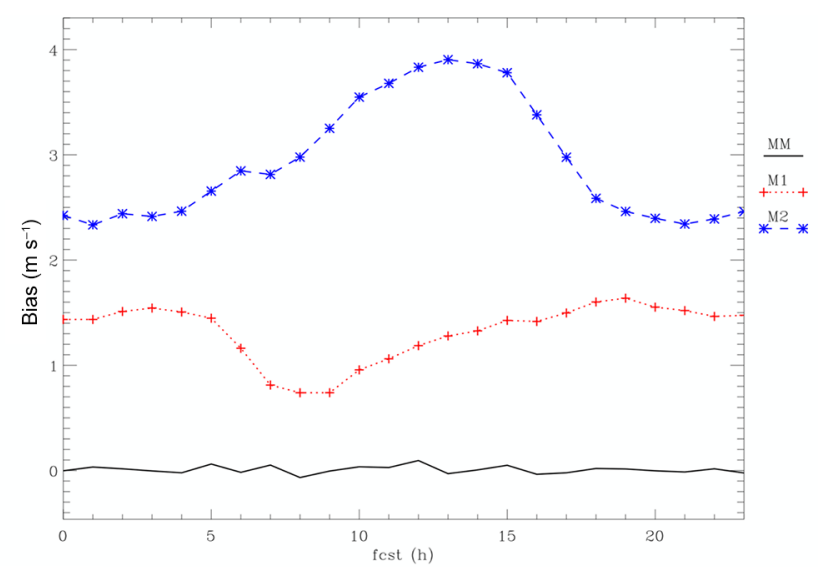

(d)

Figure 2. RMSE and BIAS for Lecce site versus forecast time (24h). M1 and M2 are the models forming the multi-model (MM). (a) RMSE for Shortwave Radiation; (b) RMSE for Wind speed; (c) BIA for Shortwave Radiation; (d) BIA for Wind speed. The difference between the RMSE of the best model and that of the MM (BEST-MM) is also shown in (a) and (b). The RMSE statistic is computed considering the 20 attempts as a whole. The boxes on the MM RMSE curve show the 25th and 75th percentile of the RMSE distribution for the 20 attempts, while the error bars extent between the maximum and minimum value of the RMSE for the 20 attempts.

\subsection{First results: RAMS and WRF performances}

Table 1 summarizes the results of the comparison between models and observations.

Initial comparisons show that the minimum hourly radiation errors (RMSE) are $96 \mathrm{~W} \mathrm{~m}^{-2}$ for Lecce and $99 \mathrm{~W} \mathrm{~m}^{-2}$ for Lamezia Terme (RAMS model). WRF performance is quite similar for both experimental sites.

Wind speed is better simulated at Lamezia Terme, where the RMSE is $1.3 \mathrm{~m} \mathrm{~s}^{-1}$ for RAMS $\left(1.4 \mathrm{~m} \mathrm{~s}^{-1}\right.$ for WRF). At Lecce, WRF errors are higher than RAMS and also higher than errors for Lamezia Terme.

For these specific runs, RAMS has slightly better performance compared to WRF and can be considered as the "best model". These results are encouraging because the errors of the models are consistent with the state-of-the-art results obtained with mesoscale models in similar studies (Tiriolo et al., 2015; Kotroni and Lagouvardos, 2004; Gomez et al., 2014).

\subsection{The MULTI-MODEL results}

Here we present the results of the multi-model technique adopted to reduce the forecast errors.

In the following these abbreviations are adopted: M1 = RAMS $; \quad$ M2 = WRF; MM = Multi Model; BEST$\mathrm{MM}=$ difference between best model and Multi Model at the specific forecasting time.

Figure 2a and $b$ show that the RMSE of the MM is lower than the RMSE of the best model (M1) for Lecce. The reduction of the error varies depending on the parameter and forecast time. For the solar radiation the error reduction is maximum in correspondence of the middle of the day, when the solar irradiance is at its maximum. Radiation er- 


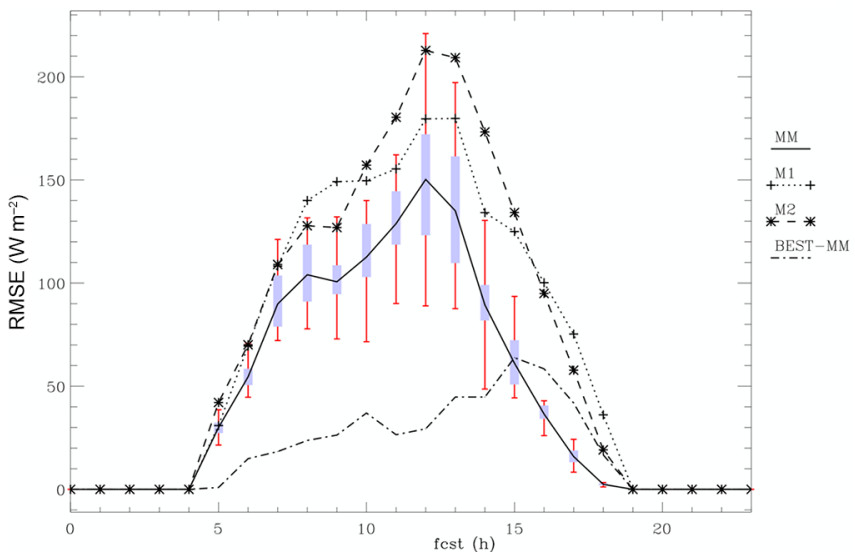

(a)

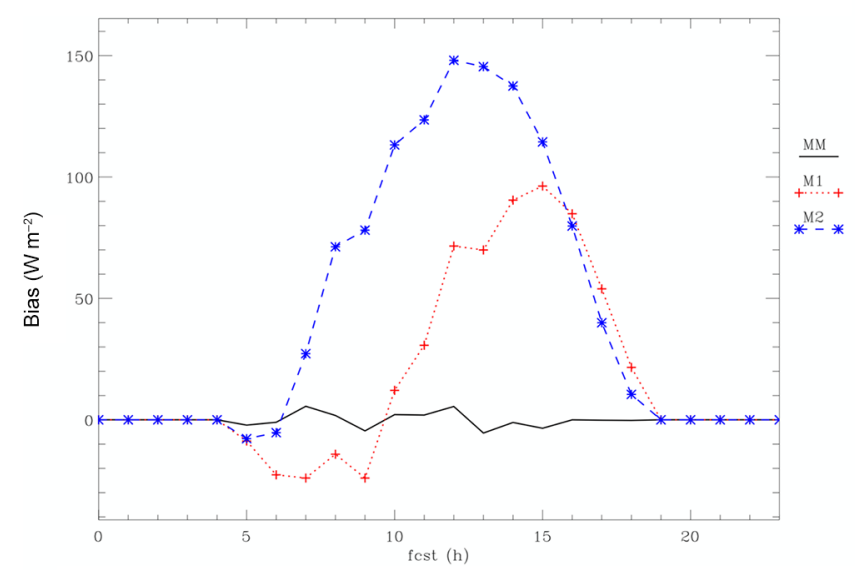

(c)

Figure 3. Same as Fig. 2 for the Lamezia Terme site.

rors are reduced up to $30 \%$ compared to the best model and, in some cases, up to $40 \%$ compared to the less accurate model. The maximum RMSE reduction occurs at 15:00 UTC $\left(60 \mathrm{~W} \mathrm{~m}^{-2}\right)$.

The Wind Speed RMSE shows a clear diurnal cycle for M2, not shown by M1. The MM lowers the RMSE of the best model up to $1.2 \mathrm{~m} \mathrm{~s}^{-1}$ (03:00 and 19:00 UTC), showing the significant impact (more than $50 \%$ reduction of the best model RMSE at 03:00 and 19:00 UTC) of the postprocessing technique on the model performance. The behaviour of the MM RMSE over the 20 attempts shows a stable behaviour and the RMSE varies by less than $0.5 \mathrm{~m} \mathrm{~s}^{-1}$ for most forecast times.

Figure $3 \mathrm{a}$ and $\mathrm{b}$ show the results for Lamezia Terme (RMSE); also for this site it is evident that the RMSE of the MM is less than the RMSE of both models. More in detail the RMSE of the short-wave radiation is reduced up to $60 \mathrm{~W} \mathrm{~m}^{-2}$ (15:00 UTC) and the MM improves the RMSE of the best model by more than $10 \%$ for most diurnal hours.

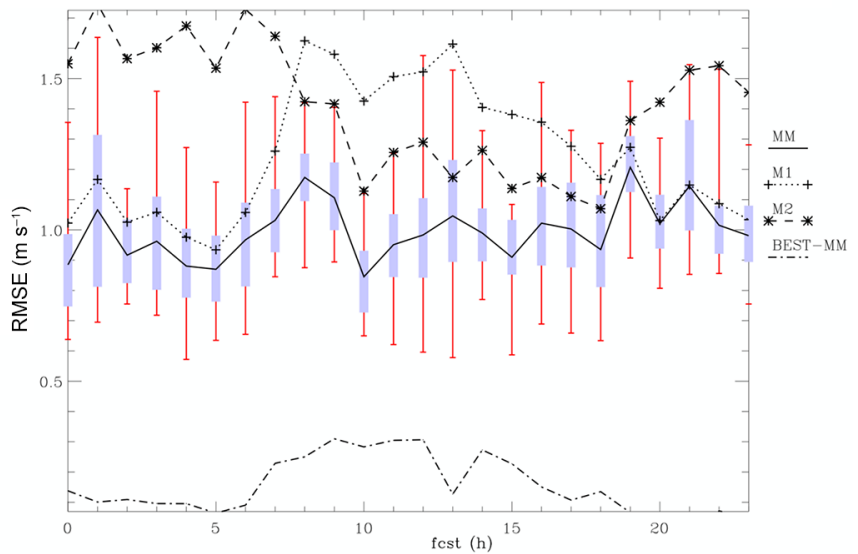

(b)

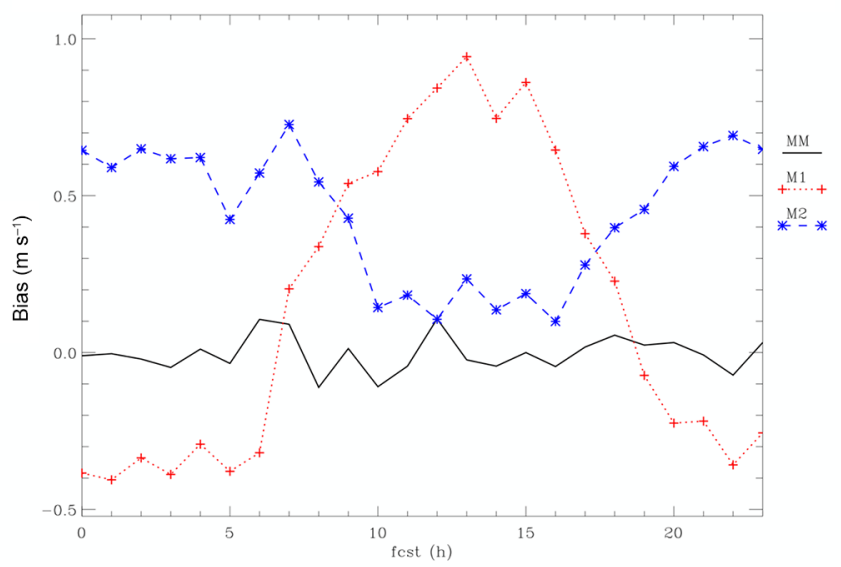

(d)

For the wind speed, the MM improvement is less evident compared to Lecce even if it is still sizable (i.e. larger than $10 \%$ of the best model RMSE) especially for the diurnal hours. Also, the variation of the MM RMSE over the 20 attempts shows a larger variability $\left(0.5-1.0 \mathrm{~m} \mathrm{~s}^{-1}\right)$ compared to Lecce.

Figure 2c and d (Lecce) and Fig. 3c and d (Lamezia Terme) show that the BIAS has been successfully removed for both parameters and sites because the BIAS of the MM is close to 0 for all forecast times. From the results of Figs. 2 and 3, it follows that the multi-model technique has been able to improve the performance of the best model, regardless to the parameter and forecast hour, showing the significant impact that this method has on the forecast for the two sites. However, the variability of the MM performance for the wind speed RMSE at Lamezia Terme may indicate the need to improve the MM performance. 


\section{Conclusive remarks}

RAMS and WRF models have good performances in predicting the shortwave radiation and wind speed, with RMSE of the order of $95 \mathrm{~W} \mathrm{~m}^{-2}$ and $1.3 \mathrm{~m} \mathrm{~s}^{-1}$, respectively.

The performance is further improved by the application of the multi-model technique for both wind speed and shortwave radiation both at Lamezia Terme and Lecce, and the RMSE is reduced by a sizeable fraction (almost always larger than $10 \%$ of the model RMSE) depending on the forecasting time and parameter.

These results are important considering the short period used for training the MM; however, the results for the Wind Speed at Lamezia Terme show a large variability, that could be likely reduced by enriching the training period (e.g. considering longer training period, different model's initializations, more members participating to the multi-model).

Acknowledgements. This work was partially supported by the project PONa3_00363 "High Technology Infrastructure for Climate and Environment Monitoring (I-AMICA)", and developed in the framework of the "EERA JP Wind" programme.

Edited by: S.-E. Gryning

Reviewed by: two anonymous referees

\section{References}

Alessandrini, A., Sperati, S., and Pinson, P.: A comparison between the ECMWF and COSMO Ensemble Prediction Systems applied to short-term wind power forecasting on real data, Appl. Energ., 107, 271-280, 2013.

Chen, C. and Cotton, W. R.: A One-Dimensional Simulation of the Stratocumulus-Capped Mixed Layer, Bound.-Lay. Meteorol., 25, 289-321, 1983.

Chou, M. D. and Suarez, M. J.: A solar radiation parameterization for atmospheric studies, NASA Tech. Memo, 104606, 15, 40 pp., 1999.

Cotton, W. R., Pielke Sr., R. A., Walko, R. L., Lista, D. E., Tremback, C. J., Jiang, H., McAnelly, R. L., Harrington, J. Y., Nicholls, M. E., Carrio, G. G., and McFadden, J. P.: RAMS 2001: Current status and future directions, Meteorol. Atmos. Phys., 82, 5-29, 2003.
Giebel, G., Brownsword, R., Kariniotakis, G., Denhard, M., and Draxl, C.: The State of the Art in Short-Term Prediction of Wind Power, Anemos.plus, deliverable report, 2011.

Gomez, I., Caselles, V., and Estrela, M. J.: Real-time weather forecasting in the Western Mediterranean Basin: An application of the RAMS model, Atmos. Res., 139, 71-84, 2014.

GSE, Rapporto Statistico "Energia da Fonti Rinnovabili in Italia - 2014", available at: http://www.gse.it/it/salastampa/GSE_ Documenti/Rapporto\%20statistico\%20GSE\%20-\%202014.pdf (last access: 19 April 2016), 2015.

Janjić, Z. I.: The Step-Mountain Eta Coordinate Model: Further developments of the convection, viscous sublayer, and turbulence closure schemes, Mon. Weather Rev., 122, 927-945, 1994.

Kotroni, V. and Lagouvardos, K.: Evaluation of MM5 HighResolution Real-Time Forecast over the Urban Area of Athens, Greece, J. Appl. Meteorol., 43, 1666-1678, 2004.

Monteiro, C., Bessa, R., Miranda, V., Botterud, A., Wang, J., and Conzelmann, G.: Wind Power Forecasting: State-of-the-Art 2009, Argonne National Laboratory ANL/DIS-10-1, November 2009.

Pinson, P., Nielsen, H. A., Madsen, H., and Kariniotakis, G.: Skill forecasting from different wind power ensemble prediction methods, J. Phys. Conf. Ser., 75, 012046, doi:10.1088/17426596/75/1/012046, 2007.

Pinson, P., Nielsen, H. A., Madsen, H., and Kariniotakis, G.: Skill forecasting from ensemble predictions of wind power, Appl. Energ., 86, 1326-1334, 2009.

Skamarock, W. C., Klemp, J. B., Dudhia, J., Gill, D. O., Barker, D., Duda, M. G., Huang, X.-Y., Powers, J. G., and Wang, W.: A Description of the Advanced Research WRF Version 3, NCAR Technical Note NCAR/TN-475+STR, doi:10.5065/D68S4MVH, 2008.

Smagorinsky, J.: General circulation experiments with the primitive equations. Part I, The basic experiment, Mon. Weather Rev., 91, 99-164, 1963.

Tiriolo, L., Torcasio, R. C., Montesanti, S., and Federico, S.: Verification of a Real Time Weather Forecasting System in Southern Italy, Adv. Meteorol., 2015, 758250, doi:10.1155/2015/758250, 2015.

Von Bremen, L.: Combination of deterministic and probabilistic meteorological models to enhance wind farm forecast, J. Phys. Conf. Ser., 75, 012050, doi:10.1088/1742-6596/75/1/012050, 2007. 